\title{
Development of a novel multiplex DNA microarray for Fusarium graminearum and analysis of azole fungicide responses
}

\author{
Rayko Becher ${ }^{1}$, Fabian Weihmann ${ }^{1}$, Holger B Deising ${ }^{1,2}$, Stefan GR Wirsel ${ }^{1,2^{*}}$
}

\begin{abstract}
Background: The toxigenic fungal plant pathogen Fusarium graminearum compromises wheat production worldwide. Azole fungicides play a prominent role in controlling this pathogen. Sequencing of its genome stimulated the development of high-throughput technologies to study mechanisms of coping with fungicide stress and adaptation to fungicides at a previously unprecedented precision. DNA-microarrays have been used to analyze genome-wide gene expression patterns and uncovered complex transcriptional responses. A recently developed one-color multiplex array format allowed flexible, effective, and parallel examinations of eight RNA samples.

Results: We took advantage of the $8 \times 15$ k Agilent format to design, evaluate, and apply a novel microarray covering the whole F. graminearum genome to analyze transcriptional responses to azole fungicide treatment. Comparative statistical analysis of expression profiles uncovered 1058 genes that were significantly differentially expressed after azole-treatment. Quantitative RT-PCR analysis for 31 selected genes indicated high conformity to results from the microarray hybridization. Among the 596 genes with significantly increased transcript levels, analyses using GeneOntology and FunCat annotations detected the ergosterol-biosynthesis pathway genes as the category most significantly responding, confirming the mode-of-action of azole fungicides. Cyp51A, which is one of the three F. graminearum paralogs of Cyp51 encoding the target of azoles, was the most consistently differentially expressed gene of the entire study. A molecular phylogeny analyzing the relationships of the three CYP51 proteins in the context of 38 fungal genomes belonging to the Pezizomycotina indicated that CYP51C (FGSG_11024) groups with a new clade of CYP51 proteins. The transcriptional profiles for genes encoding ABC transporters and transcription factors suggested several involved in mechanisms alleviating the impact of the fungicide. Comparative analyses with published microarray experiments obtained from two different nutritional stress conditions identified subsets of genes responding to different types of stress. Some of the genes that responded only to tebuconazole treatment appeared to be unique to the F. graminearum genome.

Conclusions: The novel F. graminearum $8 \times 15 \mathrm{k}$ microarray is a reliable and efficient high-throughput tool for genome-wide expression profiling experiments in fungicide research, and beyond, as shown by our data obtained for azole responses. The array data contribute to understanding mechanisms of fungicide resistance and allow identifying fungicide targets.
\end{abstract}

\section{Background}

The ascomycete Fusarium graminearum Schwabe (teleomorph Gibberella zeae (Schweinitz) Petch) is an important member of the causal agents of the Fusarium

\footnotetext{
* Correspondence: stefan.wirsel@landw.uni-halle.de

'Institut für Agrar- und Ernährungswissenschaften, Naturwissenschaftliche Fakultät III, Martin-Luther-Universität Halle-Wittenberg, Betty-Heimann-Str. 3, D-06120 Halle (Saale), Germany

Full list of author information is available at the end of the article
}

Head Blight (FHB) complex in cereals, a disease of global concern in wheat and barley [1]. Typically, spores dispersed by raindrops or wind infect flowering spikelets during humid weather at anthesis which may lead to serious FHB under post-flowering moisture conditions $[1,2]$. Like in FHB, several Fusarium species including F. graminearum are responsible for a disease on basal stem tissue of cereals, including maize, called crown rot. Beside considerable yield losses, drastic quality losses

\section{Biomed Central}


may result from contamination of grains with mycotoxins produced by $F$. graminearum. Like other members of the genus, F. graminearum produces a mixture of several mycotoxins, including deoxynivalenol (DON) that cause severe threats to human and animal health [3]. National governments have established strict regulations for tolerable mycotoxins levels in cereal products.

Azole fungicides are indispensable for the control of FHB. These fungicides inhibit the cytochrome P450 sterol $14 \alpha$-demethylase (CYP51, syn. ERG11), an enzyme that is essential for ergosterol biosynthesis, leading to disturbance of fungal membrane integrity. The current limitation of alternative fungicides to control effectively FHB has resulted in increased application of azoles. As a result, strains exhibiting increased resistance to this fungicide class were recovered from F. graminearum field populations [4,5]. Declining efficacies of azoles have also been reported in other fungal cereal pathogens, e.g. Septoria tritici and Blumeria graminis $[6,7]$. The molecular basis of fungicide resistance, however, is unclear in many cases. Fungicides currently used in agriculture only address a limited number of molecular targets, most of which are enzymes of the ergosterol biosynthetic pathway. Thus, the discovery of novel fungicides with novel modes-of-action would represent a break-through in chemical plant protection. Genomewide transcriptome analysis may help suggesting mechanisms of drug resistance [8].

The importance of $F$. graminearum for agronomy but also for basic research stimulated efforts to sequence its genome [9]. Recently, the third improved annotation of the genome (FG3) that lists 13,332 genes has become available. Gene calls from the first genome annotation by the Broad Institute (Cambridge, MA, USA) and from an annotation by the MIPS Institute (München, Germany) were combined to develop a DNA microarray using the Affymetrix GeneChip format [10]. While the Affymetrix photolithographical method allowed synthesis of short oligonucleotides ( 25 mers), the more recently developed ink jet procedure of Agilent synthesizes longer 60 mer oligonucleotides in situ which increased the detection sensitivity [11]. In addition, for Agilent several multiplex array formats are available. The Agilent $8 \times 15 \mathrm{k}$ format holds eight independent microarrays on a single slide, each carrying up to 15,000 spotted oligonucleotides, which is sufficient to cover most fungal genomes with at least one probe per gene. As demonstrated previously, the high specificity and affinity of long oligomer probes allow representing genes on microarrays by a single probe [12].

In this study, we developed a novel microarray designed for the $8 \times 15 \mathrm{k}$ Agilent multiplex format. To validate its reliability for expression profiling experiments, this array was employed to determine the genome-wide transcriptional response of F. graminearum to treatment with the sterol biosynthesis inhibitor tebuconazole. The application of recent microarray statistics and functional gene enrichment approaches uncovered the most significant responses for several sterol biosynthesis pathway genes. In addition, amongst the many genes encoding ABC transporters and transcription factors, some were identified that showed significantly altered transcript levels that were only observed in our tebuconazole study but not in a previously published microarray experiment that examined starvation stress conditions.

\section{Methods}

\section{Fungal cultivation}

In this study, we used Fusarium graminearum strain NRRL 31084 (syn. PH1). To warrant high reproducibility, conidial stock suspensions $\left(10^{6}\right.$ conidia/ml $)$ were stored in $20 \%(\mathrm{v} / \mathrm{v})$ glycerol at $-80^{\circ} \mathrm{C}$. Fungal cultivation and preparation of conidia suspension were performed as described before [13].

To prepare RNA and genomic DNA used for the microarray preselection experiment (see below), $10^{6}$ conidia were inoculated into three $300 \mathrm{ml}$ flasks containing $100 \mathrm{ml}$ complete medium (CM) [14] and cultivated at $23^{\circ} \mathrm{C}$ with shaking at $150 \mathrm{rpm}$. Mycelia were harvested after $24 \mathrm{~h}$, washed with sterile distilled water and re-inoculated into $100 \mathrm{ml}$ of CM, minimal medium (MM) [14] lacking a nitrogen source (MMN) or MM without a carbon source (MMC). After $12 \mathrm{~h}$ of cultivation at $23^{\circ} \mathrm{C}$ with shaking at $150 \mathrm{rpm}$, the mycelia were harvested, frozen in liquid nitrogen, and kept at $-80^{\circ} \mathrm{C}$ until preparation of nucleic acids. For the preparation of RNA used for microarray experiments investigating azole responses, $10^{6}$ conidia were inoculated into six 300-ml-flasks containing $100 \mathrm{ml} \mathrm{CM}$ and incubated as above. After $24 \mathrm{~h}$, three of the cultures were adjusted to $5 \mathrm{mg} / \mathrm{l}$ tebuconazole. Incubation of all cultures continued for further $12 \mathrm{~h}$, and cultures were harvested as described above.

\section{Nucleic acid isolation}

Total RNA was isolated from mycelia grown in vitro with the RNeasy Plant Mini Kit (Qiagen, Hilden, Germany) following the manufacturer's protocol. The optional on-column DNase digestion step was performed using RNase-free DNase (Qiagen). Genomic DNA was isolated from mycelium cultured in CM as described [15]. Quantity and purity of nucleic acid preparations were determined using a NanoDrop ND-1000 UV-VIS spectrophotometer (Thermo Scientific, Wilmington, USA). RNA quality was assessed with a BioAnalyzer 2100 (Agilent Technologies, Böblingen, Germany). 


\section{Microarray development}

The microarray was designed in collaboration with an Agilent-certified service provider (imaGenes, Berlin, Germany) that applied an in-house developed approach for selection of the best performing probes for each gene (http://www.imagenes-bio.de/services/microarray/ pss/mechanism). Using the annotation FG3, for each gene model, several 60 mer oligonucleotides were selected by the software eArray (https://earray.chem.agilent.com/earray/) and were then filtered by proprietary software (imaGenes). In addition to the 13,332 genes of the FG3 annotation, available cDNA sequences of the TRI (= trichothecene biosynthesis) gene cluster from nivalenol (NIV) producing strains of F. graminearum, i.e. strains H88-1 (accessions: AAK53574 to AAK53581, AAM22490) and NRRL 13383 (accessions: AAM 48747 to AAM48754) were chosen for oligonucleotide selection. The inclusion of these cDNA sequences was considered useful as genes of the TRI cluster of NIVproducing strains show several differences that might not reliably allow quantitative detection with oligonucleotides derived from DON-type PH1 sequences. Throughout this study, data from these additional cDNA-based probes were excluded from analyses.

Thereafter, up to nine oligonucleotides per gene were assembled on a pre-selection array using the Agilent $2 \times$ $105 \mathrm{k}$ format and hybridized against cyanine 3 (Cy3)labeled genomic DNA and cRNA obtained from pooled total RNA derived from three cultures grown in CM, MMN, and MMC. Based on the RNA hybridization results, for each gene the relative ratio of probes was determined that yielded significant signal intensities. In case that this ratio was $60 \%$ higher than the background, a gene was considered expressed. For these genes, final oligonucleotide selection relied on the results from RNA hybridization, whereas for genes considered nonexpressed hybridization results from genomic DNA were used. The final array design applied a proprietary algorithm (imaGenes) that used different criteria for expressed and non-expressed genes. The optimal oligonucleotides finally assembled in the $8 \times 15 \mathrm{k}$ Agilent microarray format for analyzing transcriptomic responses of F. graminearum is publicly available (Additional file 1). Microarrays can be ordered at Agilent using design ID 021301. Since this format is highly flexible in production it can easily be updated if future annotations should modify some gene models.

\section{Microarray sample preparation and hybridization}

For sample preparation and array processing the Agilent protocol "One-color microarray-based gene expression analysis (Quick Amp Labeling)" was used (http://www. chem.agilent.com). Briefly, the recommended volume of control RNAs (Agilent One-Color RNA Spike-In Kit) was added to $200 \mathrm{ng}$ of total RNA. Thereafter, Cy3labeled cRNA was produced, using the Agilent Quick Amp Kit (one-color) according to the manufacturer's protocol. Labeled cRNA was purified with the RNeasy mini kit (Qiagen). Yield and Cy3 incorporation rates were analyzed using a NanoDrop ND-1000 UV-VIS spectrophotometer. In addition, size distribution of cRNAs was assessed by a BioAnalyzer 2100. Genomic DNA was Cy3-labeled using the Genomic DNA Enzymatic Labeling Kit (Agilent) according to the manufacturer's recommendations.

Hybridization of the $105 \mathrm{k}$ pre-selection arrays was performed with either $5 \mu \mathrm{g}$ or $1.5 \mu \mathrm{g}$ of the Cy3-labeled gDNA or cRNA derived from a pooled RNA sample as described above. For hybridizations using the $15 \mathrm{k}$ arrays, 600 ng of Cy3-labeled cRNA was used. Hybridization was performed with eight cRNA samples. Six cRNA samples derived from three independent untreated and three independent azole-treated liquid cultures (see above). The remaining two samples represented additional technically repeated hybridizations that used the labeled cRNAs from one of the untreated cultures. Hybridization was set up in a Microarray Hybridization Chamber (Agilent) and was incubated in a hybridization oven (Agilent) at $65^{\circ} \mathrm{C}$ and $10 \mathrm{rpm}$ for $17 \mathrm{~h}$. After performing the suggested washing steps, slides were scanned by a microarray scanner (Agilent) at $5 \mu \mathrm{m}$ resolution.

\section{Normalization and statistical analysis of microarray data}

The microarray scan data were analyzed by Agilent's Feature Extraction software, which estimates the background level and gives a feature-specific background cut-off level per chip. The background-corrected raw signals were converted to gene signals by a median polish algorithm as implemented in the RMA (robust multi-array average) algorithm [16]. Gene signals were normalized between experiments by quantile normalization [17].

Relying on the normalized gene signals, the average $\log _{2}$ fold-changes between both experimental groups (tebuconazole-treated vs. untreated) were assessed for each gene. We applied the optimal discovery procedure (ODP) to identify significant changes in relative transcript levels [18]. This combinatorial statistical approach is available in the open-source software package EDGE version 1.1.291 [19]. The identification of differentially expressed genes includes two steps. First, ODP statistics is applied for each gene, which results in a ranking of the genes from most to least differentially expressed. In a second step, statistical significance is estimated by using the false discovery rate (FDR) methodology [20] which results in the assignment of a $q$-value for each gene. The $q$-value gives the expected rate of false 
positives amongst those genes that were assigned as differentially expressed.

Datasets of processed signal intensities from biological and technical replicates were evaluated with Pearson's coefficient of correlation (R) using MS Excel 2007 (Microsoft, Redmond, USA).

\section{Bioinformatics}

\section{Functional enrichment analyses}

Gene sets were examined for significant enrichment of functional categories using two annotation schemes, i.e. "The Functional Catalogue" (FunCat) [21] and "GeneOntology" (GO) [22]. The FunCat annotation of the F. graminearum genome was provided by the MIPS Fusarium graminearum database (FGDB) (http://mips. helmholtz-muenchen.de/genre/proj/FGDB/). We performed the GeneOntology annotation of the F. graminearum genome by subjecting all 13,332 proteins of the FG3 annotation to Blast2GO (version 2.3.6) analysis (http://www.blast2go.org). This software extracts GO terms characterizing the putative function of the proteins relying on ontologies mapped to homologous proteins identified by BLAST [23]. BlastP parameters that differed from the default were set as follows: "Number of Blast Hits" at 30, "Blast ExpectValue" at 1.0E-10. Furthermore, the "Try SIMAP first" option was chosen. The GO annotation was performed with default settings except for the "Annotation Cutoff" value that was set to 30 . Thereby, Blast $2 \mathrm{GO}$ could assign 8463 proteins to at least one of the general GO categories of biological process, molecular function, and cellular component. In analogy to FunCat enrichment analysis at the FGDB server, assessment of statistically significant GO term enrichment utilized the hypergeometric distribution function as implemented in MS Excel. The resulting $p$ values from both, the FunCat and the GO enrichment analyses, were corrected for multiple testing by applying the Benjamini-Hochberg FDR procedure [24]. The cutoff for FDR-corrected $p$ values was set to 0.05 .

\section{Identification and assignment of putative homologs}

Microarray data analyses were focused, amongst others, on genes of the ergosterol biosynthesis pathway. For this purpose, at first sequences of Saccharomyces cerevisiae proteins known to be involved in ergosterol biosynthesis [25] were retrieved from the Saccharomyces Genome Database (SGD) (http://www.yeastgenome.org/). These sequences were used as queries in the BlastP search option implemented at the FGDB server (see above) to retrieve putative homologs from the F. graminearum proteome (see Additional file 2).

We examined the phylogenetic relationships of the CYP51/ERG11 proteins from F. graminearum by comparison to the corresponding proteins of other fungi (see below). To identify such proteins in 38 currently annotated proteomes of the Pezizomycotina we used the ERG11 sequence from Saccharomyces cerevisiae as the query in BlastP searches at NCBI Genomic Blast (http:// www.ncbi.nlm.nih.gov/sutils/genom_table.cgi), at the Joint Genome Institute database (http://www.jgi.doe.gov/ genome-projects), or at the NITE Institute database (http://www.bio.nite.go.jp/dogan).

Furthermore, we assessed the phylogenetic relationships between all putative ATP-binding cassette (ABC) transporters from $F$. graminearum. The identification of such proteins relied on the FunCat and GO annotations described above. FGDB provided 78 genes annotated in the FunCat category 'ABC transporters' (20.03.25). Manual revision appeared to be necessary since this list also contained MFS (= major facilitator superfamily) transporters and other non-ABC proteins. Beyond those, we excluded four additional proteins that lacked any transmembrane domains, which were identified by the TMHMM software (http://www.cbs.dtu.dk/services/ $\mathrm{TMHMM} /$ ), resulting in a set of 50 putative $\mathrm{ABC}$ transporters. In a second analysis using the GO annotations, we applied the GO term 'ATPase activity, coupled to transmembrane movement of substances' (GO:042626). This recovered four additional proteins that were not found by FunCat and which were included in the molecular phylogeny. To assign all identified ABC transporters to one of the known subfamilies [26,27] we analyzed the distribution of predicted transmembrane and ATP binding domains by the TMHMM (see above) and the Conserved Domain Search (CDS) softwares (http://www.ncbi.nlm.nih.gov/Structure/cdd/cdd.shtml). In addition, BlastP searches using $\mathrm{ABC}$ transporters from $S$. cerevisiae and C. albicans as queries assisted in the classification of the corresponding F. graminearum proteins.

Predicted putative $F$. graminearum transcription factors were obtained from the Fungal Transcription Factor Database (FTFD) (http://ftfd.snu.ac.kr/). Transcription factors whose transcript levels differed significantly between the control and the fungicide treatment in the microarray experiments were used as queries in BlastP searches against the proteomes of $S$. cerevisiae (at SGD; see above) and Candida albicans (CGD; http://www. candidagenome.org). Vice versa, best hits were used for BlastP searches against the $F$. graminearum proteome.

\section{Phylogenetic analyses}

For phylogenetic analyses, we used the software MEGA version 4 [28]. Alignments were created by the implemented ClustalW algorithm, using the substitution BLOSUM weight matrix and the following parameters. For pairwise and multiple alignment the gap opening penalty was set to 10 , whereas the gap extension penalties were set to 0.1 , and respectively 0.05 . Evolutionary history was inferred using the Neighbor-Joining method. 
Evolutionary distances were computed using the Poisson correction method. Gaps and missing data were eliminated only from pairwise sequence comparisons (Pairwise deletion option). The confidence of the resulting tree was estimated with 1000 bootstrap iterations.

\section{Comparative analyses using published microarray data}

Microarray data analyzing in F. graminearum two nutritional stress conditions, i.e. carbon and nitrogen starvation, were published [10] and deposited at the MIAME-compliant Plant Expression Database (http:// www.plexdb.org). The RMA-normalized signal data were assigned to the updated gene names based on the ProbeSet mapping table available at the MIPS FTP sites (ftp://ftpmips.gsf.de/FGDB/) and subjected to the same statistical analyses described above. Genes determined to be differentially expressed in any of the three stress conditions, i.e. tebuconazole treatment, carbon and nitrogen starvation, were assessed for common and specific responses using MS Excel. We performed BlastP searches (http://blast.ncbi.nlm.nih.gov/Blast.cgi) at the database 'Non-redundant protein sequences (nr)' and organism 'fungi (taxid:4751)' to check whether those genes that only responded to tebuconazole were exclusively found in the F. graminearum genome.

\section{Deposition of microarray data}

MIAME-compliant information about the $8 \times 15 \mathrm{k}$ F. graminearum microarray platform and the azole treatment experiment have been deposited in the NCBI Gene Expression Omnibus (GEO) database (http://www. ncbi.nlm.nih.gov/geo) under the accession numbers GPL11158 (platform) and GSE 25114 (experiment).

\section{Quantitative RT-PCR}

qRT-PCR used the Power Sybr Green RNA-to- $C_{T}$ 1-step Kit (Applied Biosystems, Darmstadt, Germany) and the iCycler model MyiQ Single color (Bio-Rad Laboratories, München, Germany), according to the manufacturer's protocols. Reactions of $20 \mu \mathrm{l}$ contained $20 \mathrm{ng}$ of the same total RNAs that had previously been used for microarray hybridization. For all reactions, melting curve analyses at the end of the amplification and subsequent electrophoresis on $1.5 \%$ agarose gels allowed verifying amplification of a single fragment. Additionally, for each gene assessed, one reaction was purified with the SureClean Kit (Bioline, Luckenwalde, Germany) and sequenced with the BigDye Terminator v1.1 Cycle Sequencing Kit (Applied Biosystems). For each gene analyzed and each RNA preparation used, qRT-PCR was conducted in three technical repeats.

Primers were designed with the software NCBI Primer-BLAST (http://www.ncbi.nlm.nih.gov/tools/primerblast/) (Table 1). Primers for the reference genes (see below) included a predicted intron, if available, which tested for contamination of RNA preparations with genomic DNA. In case of FGSG_10791, a predicted intron could not be confirmed.

Analysis of qRT-PCR data applied the sigmoidal curve-fitting method [29] that calculates the initial fluorescence $\left(R_{0}\right)$, which relates to the initial amount of the target in a sample. Computation of $R_{0}$ was conducted using the software Sigmaplot version 11 (Systat Software, Erkrath, Germany). As quantification of gene expression requires normalization by stable internal references, a minimum of three reference genes is recommended to derive a combined normalization factor used to adjust the data of the genes of interest [30]. To identify genes with putatively stable expression levels, normalized signals from all hybridizations of the $8 \times 15 \mathrm{k}$ microarray data except those from the technical replications were used to calculate a coefficient of variation $(\mathrm{CV}=\mathrm{SD} /$ mean $)$ for each gene. The least variable genes with $\mathrm{CV}<0.05$ were examined for a putative housekeeping function, according to the gene descriptions at the FGDB. As a result, FGSG_06245 (cofilin), FGSG_01244 (pre-mRNA splicing factor), and FGSG_10791 (RNA helicase) were chosen as potential reference genes. Subsequently, qRT-PCR expression data for the reference genes were subjected to analysis by the software geNORM (http://medgen.ugent.be/ jvdesomp/ genorm/) to check their expression stability [30]. All three selected reference genes yielded values for the gene-stability measure $M$ below the threshold $(M=0.7)$ and were therefore used to derive the specific normalization factor (NF) for each RNA sample as reported [30]. Finally, for each gene-of-interest the mean $R_{0}$ of the RNAs from treated and untreated mycelia were calculated and used to determine the $\log _{2}$ fold-change between the control and treatment.

\section{Results \\ Design and validation of an $8 \times 15 \mathrm{k}$ microarray for F. graminearum}

In this study, a new Fusarium graminearum microarray was designed for the $8 \times 15 \mathrm{k}$ Agilent multiplex format. The representation of most genes by a single oligonucleotide probe on the array demanded a thorough preselection. Potentially suitable 60 mer probes were determined by bioinformatics and then experimentally validated for hybridization-signal quality by a pre-selection microarray experiment. For $57 \%$ of the genes four to seven probes were assembled on the pre-selection array whereas $41 \%$ of the genes were represented by eight to nine probes. It was not possible to derive more than three probes for about $2 \%$ of the genes. For two of the annotated genes, FGSG_08274 and FGSG_12303, no specific oligonucleotide was identified, due to sequence duplication and repetitive sequence structure. 
Table 1 Primers used for qRT-PCR

\begin{tabular}{|c|c|c|c|}
\hline Gene ID $^{a}$ & Forward primer $\left(5^{\prime}-3^{\prime}\right)$ & Reverse primer $\left(5^{\prime}-3^{\prime}\right)$ & Amplicon (bp) \\
\hline \multicolumn{4}{|l|}{ CYP51 } \\
\hline FGSG_01000 & TCTACACCGTTCTCACTACTCC & GCTTCTCTTGAAGTAATCGC & 170 \\
\hline FGSG_04092 & CCCTTACACGATCACTACAGAC & CTCTTCGTTCCTITAGACACAG & 186 \\
\hline FGSG_11024 & ACTTGGTCTCACCACAGATTC & CATAGATCGGACTTCGTTTC & 187 \\
\hline \multicolumn{4}{|c|}{$A B C$ transporters } \\
\hline FGSG_00046 & CTACTATCTGGGTGAACCTGTG & GCCACTGTITTCAGGAGTATC & 209 \\
\hline FGSG_02786 & CTTCGGCAAGATATGAGCTTC & GCGAGTATGGAACTCGATAGG & 161 \\
\hline FGSG_03032 & CCTCGCAAGACAACCATACAG & AGAGTGATGTCGCGAATATCG & 176 \\
\hline FGSG_03882 & GAACTCACGTACTCTCCTCAAC & TCGACATCAACTCTCTCTTGAC & 168 \\
\hline FGSG_05076 & GTGCTATGCTCCAGCAATACC & CGATCAGAAGAGCGAACTGAG & 198 \\
\hline FGSG_06771 & TCGTCAGTCCAGAAGTCAAG & TTCGGTTCACAAAGTAGTCC & 180 \\
\hline FGSG_06881 & AAACCCCGTTGAAACAGAAC & ATCAAACCATAAGCGACGTTC & 186 \\
\hline FGSG_07325 & CCTCTATGCTITCCTTCATC & ATACCGTGTCTTCAACTGTCTC & 207 \\
\hline FGSG_08308 & ACAGGCTCGTCTATTCACAG & GACCCCTTGATAACCTCTAAC & 187 \\
\hline FGSG_08309 & GTCTCTITGTATCAGGCTTCC & САTATTCTCTCTCCCACTCATC & 199 \\
\hline FGSG_08312 & GATCGTCCTCTTCACTATTACC & CTCTCAGCAGTAACCATAGGAC & 183 \\
\hline FGSG_08373 & CTGTGACGATACCGAGCTGAC & CCTGTATCGAGCCAAATCAAG & 120 \\
\hline FGSG_08830 & GACTACTTGGCTTCTCTITCC & CACCATCTTCTTGACTACCAAC & 250 \\
\hline FGSG_10995 & ATTTATGACCACGCAGTCTGG & CTGCGAAAAGAGGTTCGTTAC & 121 \\
\hline FGSG_11028 & GACACACTACCGACACAACTG & GACTGAGAAGACGAGAAGAAG & 225 \\
\hline FGSG_11988 & GATGTAATGCTACCTGGAACAC & GATGAGACCGATTGTGAGAAC & 195 \\
\hline \multicolumn{4}{|c|}{ Transcription factors } \\
\hline FGSG_00069 & GAATCTTCTCCGGCACTCAG & TTCTTCACTTCCTTCCGAGATC & 139 \\
\hline FGSG_01293 & CCCGATATAGTCGAGCCTAAC & GGTATTGTTGCTCCGTTGAC & 101 \\
\hline FGSG_01341 & ATTCTGGCATGGATGATGAAG & CACCAAAGTCACTGGCATATCC & 162 \\
\hline FGSG_01669 & TTCGACTTCTCACAGCTCAGC & TGCCTGCATGTTGTACTGGTC & 120 \\
\hline FGSG_05949 & TTCGGCAACCATCACTCCTAG & TGCTGTTCGTTCTCTCGCACT & 100 \\
\hline FGSG_06324 & ACAGAGTGTCGAACCAGCAAC & GGTTCTCGTGGGATGCTATC & 173 \\
\hline FGSG_06810 & ATACCCGTCTCATGAACATCG & TCGATAACAGCCTTGGCTATG & 127 \\
\hline FGSG_09333 & CACAGCTCAACGATGCAATG & CAAGATAAGCAAGGATGCTGTG & 101 \\
\hline FGSG_09349 & GCTTGATTGCCCCTCTGAGA & GGTTCGCATTCTCCTGGTTC & 123 \\
\hline FGSG_10470 & AGAGATCACCACGTCCGAAC & TGGTGGGTGTAGATGTGGTTC & 114 \\
\hline FGSG_10914 & CATCGGGCTCTAGTACGAATC & CCAGAAAGCAGCAGTATGCTC & 141 \\
\hline FGSG_11561 & ACTCGAAAGCATTGGATCAGC & AGACGGACGAAATCATCGTC & 130 \\
\hline
\end{tabular}

a The given gene ID is the entry number of the F. graminearum genome annotation FG3 provided by Broad Institute.

Accordingly, 103,747 probes were assembled on a $2 \times$ $105 \mathrm{k}$ Agilent microarray that was hybridized to Cy3labeled cRNA derived from a pool of RNAs from three cultures using different media. The second hybridization used labeled genomic DNA. Transcripts were detected for about $80 \%$ of the genes when applying a proprietary threshold adjustment (imaGenes, Berlin, Germany) to the processed signal data from the RNA hybridization (data not shown). Those 10,622 genes were considered to be expressed. A proprietary algorithm (imaGenes) selected the optimal oligonucleotides for the final $15 \mathrm{k}$ array by using the results from the RNA hybridization for the expressed genes. For the 2725 genes that were not expressed, the signal data from the DNA hybridization were utilized. The final $15 \mathrm{k}$ array design comprised
15,208 F. graminearum-specific and 536 additional oligonucleotides, including internal controls (Additional file 1). In total 11,476 genes and 10 ESTs that were derived from NIV-type TRI cluster genes were represented by one oligonucleotide, whereas for 1854 genes and 7 NIV-type TRI cluster ESTs two probes were chosen.

The general quality of the signals was assessed with regard to the background noise. For all hybridizations, the average percentage of spot data determined by the Feature Extraction Software to be positive and significantly above background was $96.1 \pm 2.2 \%$. The chosen one-color hybridization method is a rather recent development for the Agilent platform. Therefore, the performance of the array was evaluated by technical 
replications that used a single RNA sample from one of the untreated cultures for three hybridization experiments to determine variation arising during all stages of data generation. Pearson's correlation coefficients (R) of $0.992,0.995$, and 0.995 ( $p<0.0001$ each) indicated highly significant conformity. In comparison, the biological replicates of the untreated and the treated samples also showed strong correlation, with $\mathrm{R}=0.939,0.946$, 0.952 , and respectively $0.945,0.958,0.968(p<0.0001$ each). The minor variations detected in these experiments demonstrated high experimental reproducibility.

\section{Gene expression in response to tebuconazole treatment}

To validate the suitability of the microarray to suggest fungicide target(s) and mode(s) of action, F. graminearum strain PH1 was exposed to $5 \mathrm{ppm}$ of tebuconazole in liquid culture for $12 \mathrm{~h}$. This concentration was previously determined as sub-lethal but strongly inhibitory for fungal vegetative growth (about 95\% on fungicide-amended agar-plates compared to untreated controls; data not shown).

Based on the processed, normalized gene signals the average $\log _{2}$ fold-change ( $\log _{2} \mathrm{FC}$ ) between both experimental groups (tebuconazole treated vs. untreated control) was assessed for each gene (Additional file 3). Statistical analysis using the optimal discovery procedure (ODP) as implemented in the EDGE software resulted in a list ranking genes according to significance based on $q$ values (Additional file 3). To identify genes that appeared differentially expressed after fungicide treatment, we applied two conditions, i.e. a cut-off for statistical significance $(q<0.04)$ and a cut-off for foldchanges $\left(\log _{2} \mathrm{FC} \geq 1\right.$ and $\left.\leq-1\right)$. This procedure identified 596 genes with significantly increased and 462 genes with significantly decreased transcript abundances. The 15 most significantly differentially expressed genes with either an increase or a decrease in response to the fungicide are listed in Table 2. Among those with increased transcript levels were five genes (FGSG_04092, FGSG_09764, FGSG_03686, FGSG_13888, and FGSG_02771) that are putatively involved in sterol metabolic processes. Among the genes exhibiting decreased transcript levels were genes that affiliated with amino acid transport (FGSG_02499, FGSG_02950, and FGSG_09354) and amino acid metabolism (FGSG_10119, FGSG_02349, FGSG_00296, and FGSG_09647).

Two functional annotation approaches, "The Functional Catalogue" (FunCat) and "GeneOntology" (GO), were used to determine whether distinct functional groups of proteins were overrepresented within the differentially expressed genes. Among the genes with increased transcript levels, these programs identified only few functional categories as significantly enriched. These analyses revealed "sterol biosynthetic process" and "tetracyclic and pentacyclic triterpenes (cholesterin, steroids and hopanoids) metabolism" as the most significantly enriched functional classes (Additional file 4). Furthermore, both analyses also identified isoprenoid metabolism and heme binding protein functions as significantly enriched. The two methods yielded distinct results for some additional categories. Whereas FunCat found protein degradation as significantly enriched, GO regarded proteins with hexosyl transferase activity and proteins involved in membrane processes and mycelium development as significantly enriched among the genes with increased transcript levels. The analyses of the genes with significantly decreased transcript levels retrieved a higher number of enriched categories. Both methods identified several central cellular processes, e.g. respiratory chain and energy generation and metabolism of amino acids and cofactors (Additional file 4).

\section{Analyses of selected gene subsets}

Previous studies on azole response and/or resistance in human pathogens to azoles have implicated a general importance of genes encoding ergosterol biosynthesis proteins, $\mathrm{ABC}$ transporters and transcription factors. However, in plant pathogens corresponding information is scarce. Therefore, we examined these genes in more detail.

\section{Ergosterol biosynthesis}

Both functional enrichment analyses uncovered proteins belonging to sterol or steroid biosynthesis as the category exhibiting the most significant response to tebuconazole treatment. This was reflected by significantly increased transcript levels of most of the genes with homologs among the ergosterol biosynthesis genes from S. cerevisiae (Additional file 2).

In contrast to $S$. cerevisiae, certain Erg genes are represented by more than one copy in some Pezizomycotina, including F. graminearum [31]. Each of the $S$. cerevisiae genes Erg3, Erg5, Erg6, Erg10, and Erg24 corresponded to two genes in the genome of F. graminearum. Interestingly, three genes, i.e. FGSG_01000, FGSG_04092 and FGSG_11024, encoded ERG11 (syn. CYP51) the molecular target of azole action. Therefore, we analyzed the evolution of CYP51 in 38 species of the Pezizomycotina that have annotated proteomes. In total, 17 of the species had one and 14 had two Cyp51 genes. The genomes of five species, i.e. F. graminearum (teleomorph Gibberella zeae), Fusarium solani (teleomorph Nectria haematococca), Aspergillus terreus, A. flavus, and $A$. oryzae, harbor three putative Cyp51 genes. We created a molecular phylogeny to examine the relationships between the CYP51 proteins from Pezizomycotina and included $S$. cerevisiae as the outgroup (Figure 1). These analyses identified three clades for the CYP51 proteins of the Pezizomycotina, two of which had 
Table 2 Genes with most significantly altered transcript levels

\begin{tabular}{|c|c|c|c|}
\hline Gene ID $^{a}$ & Annotation $^{\mathrm{b}}$ & $\log _{2} F C^{c}$ & $q^{d}$ \\
\hline & 15 most significantly increased & & \\
\hline FGSG_04092 & probable cytochrome P450 51 (eburicol 14 alpha-demethylase) & 7.35 & 0.006 \\
\hline FGSG_02255 & conserved hypothetical protein & 6.57 & 0.006 \\
\hline FGSG_02748 & related to endothelin-converting enzyme 1 & 2.80 & 0.012 \\
\hline FGSG_09764 & related to phosphomevalonate kinase & 3.33 & 0.012 \\
\hline FGSG_03686 & probable cytochrome P450 (involved in C-22 denaturation of the ergosterol side-chain) & 3.69 & 0.012 \\
\hline FGSG_04492 & conserved hypothetical protein & 4.61 & 0.012 \\
\hline FGSG_10490 & conserved hypothetical protein & 3.46 & 0.012 \\
\hline FGSG_10170 & conserved hypothetical protein & 1.80 & 0.012 \\
\hline FGSG_02721 & conserved hypothetical protein & 1.45 & 0.012 \\
\hline FGSG_13888 & related to emopamil-binding protein & 7.35 & 0.012 \\
\hline FGSG_03489 & conserved hypothetical protein & 4.35 & 0.012 \\
\hline FGSG_04874 & conserved hypothetical protein & 6.78 & 0.012 \\
\hline FGSG_05739 & conserved hypothetical protein & 6.94 & 0.013 \\
\hline FGSG_04034 & conserved hypothetical protein & 3.97 & 0.013 \\
\hline \multirow[t]{2}{*}{ FGSG_02771 } & probable KES1 - involved in ergosterol biosynthesis & 3.05 & 0.013 \\
\hline & 15 most significantly decreased & & \\
\hline FGSG_02499 & related to amino acid transport protein & -1.49 & 0.012 \\
\hline FGSG_06437 & related to triacylglycerol lipase $\vee$ precursor & -2.18 & 0.012 \\
\hline FGSG_13414 & conserved hypothetical protein & -2.13 & 0.012 \\
\hline FGSG_00788 & conserved hypothetical protein & -2.12 & 0.016 \\
\hline FGSG_10119 & related to threonine dehydratase & -2.06 & 0.027 \\
\hline FGSG_02349 & probable tyrosine-tRNA ligase & -2.33 & 0.029 \\
\hline FGSG_02950 & related to neutral amino acid permease & -2.20 & 0.029 \\
\hline FGSG_09392 & conserved hypothetical protein & -1.04 & 0.029 \\
\hline FGSG_09354 & probable neutral amino acid permease & -2.16 & 0.029 \\
\hline FGSG_04215 & related to monocarboxylate transporter 2 & -3.20 & 0.032 \\
\hline FGSG_00296 & probable ILV1 - anabolic serine and threonine dehydratase precursor & -1.94 & 0.033 \\
\hline FGSG_10809 & conserved hypothetical protein & -1.75 & 0.033 \\
\hline FGSG_08394 & conserved hypothetical protein & -1.38 & 0.033 \\
\hline FGSG_10626 & related to phenazine biosynthesis protein phz & -2.16 & 0.033 \\
\hline FGSG_09647 & probable histidinol-phosphate transaminase & -2.17 & 0.033 \\
\hline
\end{tabular}

a The given gene ID is the entry number of the F. graminearum genome annotation FG3. ${ }^{\mathrm{b}}$ Annotation as provided by the MIPS Fusarium graminearum genome database (FGDB). ${ }^{c}$ The relative transcriptional differences between fungicide-treatment vs. untreated control given as log ${ }_{2}$ transformed fold-changes. ${ }^{d}$ Statistical assessment of transcriptional differences by ODP. The $q$ value is a measure of the false-positive discovery rate in a given set of genes.

previously been termed CYP51A and CYP51B [32]. Species that had only one copy of CYP51 were exclusively found in clade B. In those species that encoded two or three CYP51 proteins one variant clustered in clade B, the other in clade A, except for Cochliobolus heterostrophus and Talaromyces stipitatus. The former possessed two CYP51B variants, whereas the latter showed a distinct CYP51 type that positioned at an unsupported branch. For species that encoded three CYP51 proteins in their genomes two patterns of clustering were observed for the third additional copy. In Aspergillus spp., the third CYP51 variants clustered either with clade A (A. flavus and A. oryzae) or with clade B (A. terreus). In contrast, the third CYP51 variants of $F$. graminearum (FGSG_11024) and F. solani formed a distinct and supported clade termed CYP51C.
Regarding the unique phylogenetic distribution of the three Cyp51 genes in F. graminearum, we analyzed whether they exhibited different transcriptional responses after azole treatment. qRT-PCR confirmed that the transcript levels of all three Cyp51 genes were significantly increased by the treatment (Table 3 ). The largest increase was observed for FGSG_04092 (= Cyp51A) that was also the most consistently differentially expressed gene of the microarray study (Table 2). The sigmoidal model applied to evaluate real-time kinetics allowed calculating the $\mathrm{R}_{0}$ value which reflects initial transcript abundance [33]. These comparisons revealed similar transcript abundances for FGSG_01000 $(=$ Cyp51B) and FGSG_11024 (= Cyp51C) in untreated mycelia. Transcript abundances of these genes were about 10 times higher than that of FGSG_04092 (= 


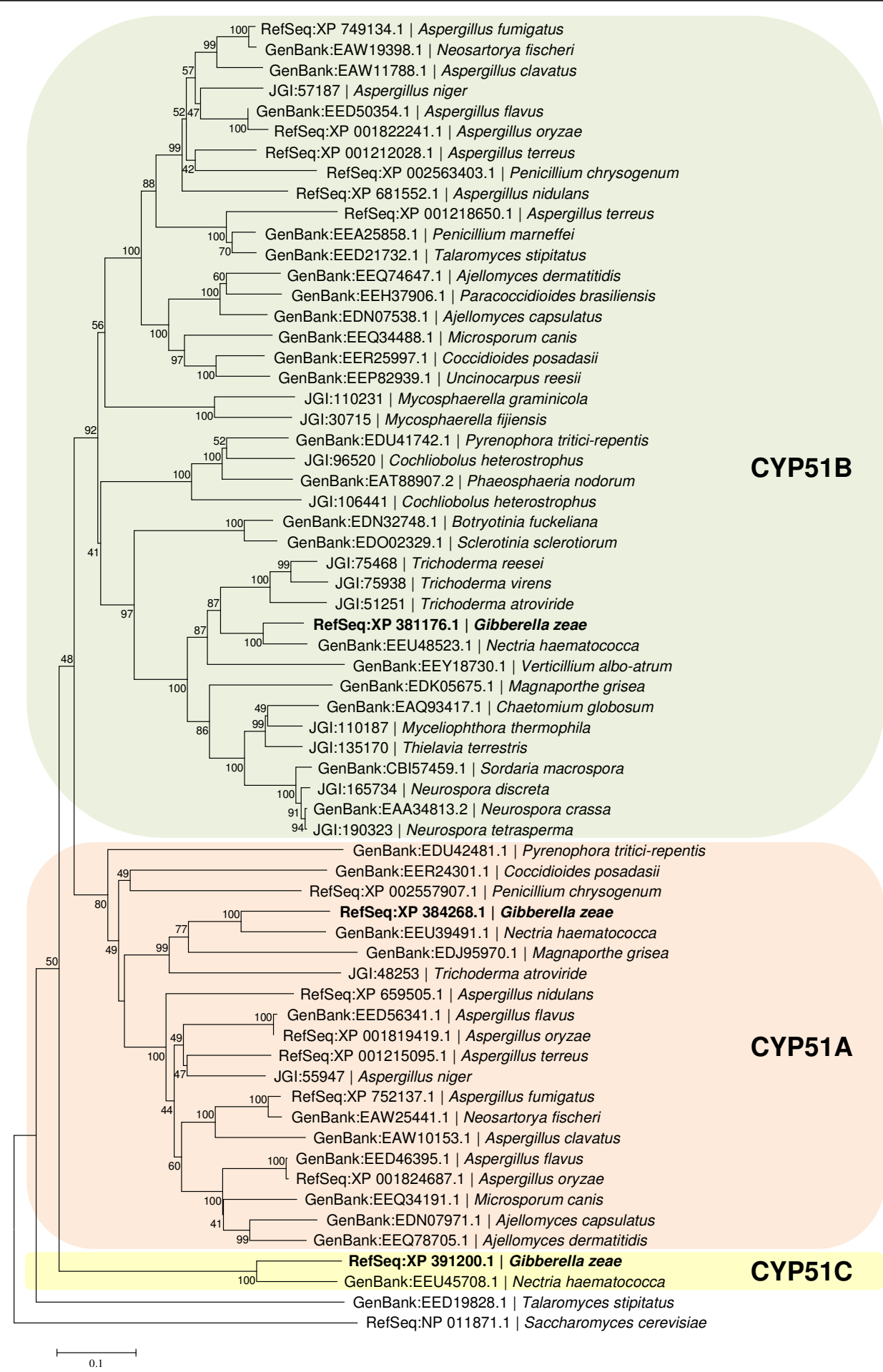

Figure 1 Molecular phylogeny of CYP51 proteins of Pezizomycotina. The depicted phylogram was obtained by Neighbor-Joining using MEGA4 software and reflects the relationships between 64 amino acid sequences of CYP51 (syn. ERG11) like proteins extracted from 39 annotated proteomes. Results from bootstrapping with 1000 replicates are indicated when higher than 30\%. 
Table 3 Comparison of differential gene expression as determined by microarray hybridization and qRT-PCR

\begin{tabular}{|c|c|c|c|c|c|}
\hline \multirow[t]{2}{*}{$\overline{\text { Gene ID }}^{\mathrm{a}}$} & \multirow[t]{2}{*}{ Type $^{b}$} & \multicolumn{2}{|c|}{ Microarray } & \multicolumn{2}{|c|}{ qRT-PCR } \\
\hline & & $\log _{c} F C$ & $q^{d}$ & $\log _{c} F C$ & $p^{\mathrm{e}}$ \\
\hline \multicolumn{6}{|l|}{ CYP51 } \\
\hline FGSG_01000 & CYP51B & 2.96 & 0.0303 & 2.89 & $<0.0001$ \\
\hline FGSG_04092 & CYP51A & 7.35 & 0.0061 & 6.92 & $<0.0001$ \\
\hline FGSG_11024 & CYP51C & 2.06 & 0.0385 & 1.54 & 0.0001 \\
\hline \multicolumn{6}{|c|}{$\mathrm{ABC}$ transporters } \\
\hline FGSG_00046 & MRP & 3.00 & 0.0392 & 1.17 & 0.0231 \\
\hline FGSG_02786 & MDR & 2.16 & 0.0392 & 1.64 & 0.0036 \\
\hline FGSG_03032 & $\begin{array}{l}\text { MDR } \\
\text { (half) }\end{array}$ & -1.29 & 0.0392 & -0.99 & 0.0793 \\
\hline FGSG_03882 & PDR & -3.65 & 0.0516 & -3.27 & 0.0560 \\
\hline FGSG_05076 & PDR (half) & 1.94 & 0.0385 & 1.17 & 0.0249 \\
\hline FGSG_06771 & MDR & 2.02 & 0.0392 & 1.56 & 0.0017 \\
\hline FGSG_06881 & MDR & 1.38 & 0.0401 & 2.08 & 0.0303 \\
\hline FGSG_07325 & MRP & 1.57 & 0.0539 & 1.42 & 0.0033 \\
\hline FGSG_08308 & MRP & 4.07 & 0.0508 & 3.41 & 0.0310 \\
\hline FGSG_08309 & PDR & -2.95 & 0.0509 & -2.05 & 0.0686 \\
\hline FGSG_08312 & PDR & 2.67 & 0.0385 & 1.90 & $<0.0001$ \\
\hline FGSG_08373 & n.d. & 1.55 & 0.0395 & 1.32 & 0.0036 \\
\hline FGSG_08830 & PDR & 6.58 & 0.0523 & 3.78 & 0.0226 \\
\hline FGSG_10995 & MRP & 2.61 & 0.0497 & 1.30 & 0.0176 \\
\hline FGSG_11028 & MRP & 1.44 & 0.0502 & -0.85 & 0.4518 \\
\hline FGSG_11988 & MDR & -1.91 & 0.0509 & -1.63 & 0.0264 \\
\hline \multicolumn{6}{|c|}{$\begin{array}{l}\text { Transcription } \\
\text { factors }\end{array}$} \\
\hline FGSG_00069 & Zn2Cys6 & -2.14 & 0.0385 & -1.81 & 0.0207 \\
\hline FGSG_01293 & Zn2Cys6 & 2.08 & 0.0385 & 1.91 & 0.0010 \\
\hline FGSG_01341 & $\mathrm{C} 2 \mathrm{H} 2$ & 1.62 & 0.0357 & 1.92 & 0.0042 \\
\hline FGSG_01669 & Zn2Cys6 & 3.86 & 0.0392 & 3.30 & 0.0002 \\
\hline FGSG_05949 & WING & 4.16 & 0.0385 & 3.96 & $<0.0001$ \\
\hline FGSG_06324 & Zn2Cys6 & 1.64 & 0.0385 & 1.00 & $<0.0001$ \\
\hline FGSG_06810 & Zn2Cys6 & 1.70 & 0.0387 & 1.35 & 0.0002 \\
\hline FGSG_09333 & Zn2Cys6 & -1.22 & 0.0385 & -0.57 & 0.0121 \\
\hline FGSG_09349 & Zn2Cys6 & -2.16 & 0.0385 & -0.98 & 0.0092 \\
\hline FGSG_10470 & $\mathrm{C} 2 \mathrm{H}_{2}$ & 4.74 & 0.0385 & 4.32 & $<0.0001$ \\
\hline FGSG_10914 & Zn2Cys6 & -1.03 & 0.0392 & -1.14 & 0.0738 \\
\hline FGSG_11561 & Zn2Cys6 & 1.48 & 0.0387 & 1.57 & 0.0162 \\
\hline
\end{tabular}

${ }^{a}$ The given gene ID is the entry number of the $F$. graminearum genome annotation FG3. ${ }^{\text {b }}$ Classification to gene subfamilies. ${ }^{\mathrm{c}}$ The relative transcriptional differences between fungicide-treatment vs. untreated control given as $\log _{2}$ transformed fold-change as determined by microarray hybridization and by qRT-PCR. ${ }^{\mathrm{d}}$ Statistical assessment of transcriptional differences by ODP. The $q$ value is a measure of the false-positive discovery rate in a given set of genes. ${ }^{e}$ Statistical assessment of transcriptional differences by Student's t-test.

ALDp, adrenoleukodystrophy protein; CYP51, cytochrome P450 sterol $14 \alpha-$ demethylase; MDR, multi drug resistance proteins; MRP, multidrug resistanceassociated proteins; PDR, pleiotropic drug resistance proteins; $\mathrm{C} 2 \mathrm{H} 2$, Cys-CysHis-His motif zinc finger proteins; WING, winged helix-turn-helix DNA-binding protein; Zn2Cys6, Zn(II)2-Cys6 binuclear cluster domain-type transcription factors; n.d. not defined.
Cyp51A) (Additional file 5). However, the response of the latter gene towards tebuconazole treatment appeared as most drastic, showing a 121-fold increase of transcript abundance.

$A B C$ transporters Previous studies on azole responses in other fungi had indicated a contribution of $A B C$ transporters to drug tolerance [34]. Using FunCat and Blast2GO annotations, 54 putative $\mathrm{ABC}$ transporter proteins were identified in the $F$. graminearum proteome. We constructed a phylogenetic tree to examine their relationships especially in the context of subfamilies known from ABC transporters [26,27] (Figure 2). Thereby, we classified multidrug resistance proteins (MDR), multidrug resistance-related proteins (MRP), pleiotropic drug resistance proteins (PDR), and adrenoleukodystrophy protein (ALDp)-type ABC transporters, which were represented by $16,16,19$, and 2 members, respectively (Figure 2 ). One putative $A B C$ transporter gene, FGSG_08373, did not group with any of these subfamilies established first in the Saccharomycotina. Its domain organization corresponded to MDR-type proteins, but it had highest similarity to PDR-type proteins from C. albicans and S. cerevisiae. Typically, ABC transporters comprise two transmembrane and two nucleotide-binding domains. However, for the MDR- and PDR-types, half-sized proteins with only one copy of the domains are also known. In F. graminearum, six halfsize MDR and three PDR-proteins exist (Figure 2). Two sequences (FGSG_08027 and FGSG_09611) seemed to miss parts of the corresponding proteins putatively as a result of erroneous ORF predictions.

The microarray analyses indicated that transcript levels of 23 genes of the $A B C$ superfamily showed more than twofold $\left(\log _{2} \mathrm{FC} \geq 1\right.$ and $\left.\leq-1\right)$ changes after tebuconazole treatment, and seven that additionally met our significance threshold of $q<0.04$ (Additional file 2). Of these, four belonged to the MDR-, two to the PDR-, and one to the MRP-type. One gene encoding a half-size MDR protein exhibited significantly reduced transcript levels. However, results from microarray analyses of gene families like the $A B C$ transporters might be blurred by mispriming due to extended sequence similarities. Therefore, qRT-PCR was performed for $16 \mathrm{ABC}$ transporter genes to evaluate the reliability of the microarray data (Table 3). For 15 of the selected genes the $\log _{2}$ FC values for tebuconazole treatment obtained by the microarray experiment were coherent with those of qRT-PCRs. For one gene, i.e. FGSG_11028, qRT-PCR did not confirm the microarray data. The relative transcript levels of different $A B C$ transporter genes, as determined by qRT-PCR varied by up to a factor of 538 




Figure 2 Molecular phylogeny of $\boldsymbol{F}$. graminearum ABC transporters. The depicted phylogram was obtained by Neighbor-Joining using MEGA4 software and reflects the relationships between 54 amino acid sequences of putative ABC transporters that were extracted from the F. graminearum proteome. Results from bootstrapping with 1000 replicates are indicated when higher than $30 \%$. Classification for subfamily uses a published nomenclature [26,27] and is indicated by colored boxes. ALDp, adrenoleukodystrophy protein; MDR, multi drug resistance proteins; MRP, multidrug resistance-associated proteins; PDR, pleiotropic drug resistance proteins; NBD, nucleotide-binding domain; TMS, transmembrane spanning domain. 
in untreated mycelia (see FGSG_02786 vs. FGSG_06771). In mycelia treated by tebuconazole, up to 778 fold differences in transcript abundances were detected (FGSG_11028 vs. FGSG_06771) (Additional file 5).

Transcription factors The microarray data were also analyzed to identify potential regulators involved in azole-induced transcriptional responses. According to the Fungal Transcription Factor Database, the F. graminearum proteome contained 660 putative transcription factors classified into 44 families (Additional file 2). The transcript levels of 22 of these genes were significantly increased, whereas 15 were decreased by the tebuconazole treatment $\left(\log _{2} \mathrm{FC} \geq 1\right.$ and $\left.\leq-1 ; q<0.04\right)$. BlastP analyses using the proteins of the increased genes as queries against the annotated proteomes of S. cerevisiae and $C$. albicans yielded hits with some proteins known to be involved in azole-stress responses in these yeasts (see Additional file 2). The proteins encoded by FGSG_06324, FGSG_10364, and FGSG_11561 resembled the sterol regulatory binding protein UPC2, which is a transcription factor controlling ergosterol biosynthetic and sterol uptake genes [35]. The protein encoded by FGSG_06810 was similar to the transcription factor TAC1, which is a regulator of $A B C$ transporters [36]. Other up-regulated transcription factors appeared to be involved in rather general stress responses. FGSG_01341, FGSG_10470, and FGSG_13711 were similar to CRZ1, a transcription factor involved in calcineurin- and $\mathrm{Ca}^{2+} /$ calmodulin-dependent signaling [37]. The proteins encoded by FGSG_09019, FGSG_01293, FGSG_01936, and FGSG_13828 are putative regulators of metabolic processes, which may be indirectly compromised by fungicide treatment. These genes exhibit similarities to yeast's transcription factors involved in phosphate metabolism, carbohydrate metabolism, and fatty acid degradation (see Additional file 2).

Among the 13 putative transcription factors, which exhibited significantly reduced transcript levels after tebuconazole treatment, FGSG_09333 showed similarity to RDR1 of $S$. cerevisiae [38]. RDR1 is a repressor of the ABC transporter gene $P d r 5$ encoding an efflux pump which is important for multidrug resistance in yeast. FGSG_12970 was similar to the transcription factor RIM101 described as a positive [39] and negative [40] regulator protein contributing to cell wall assembly and altered lipid asymmetry signaling in yeast. Due to their homologies to the yeast proteins DAL81 and ARG81, the $F$. graminearum proteins encoded by FGSG_10914 and FGSG_00144 might be involved in regulation of catabolic processes.

qRT-PCR analyses were performed for 12 genes encoding transcription factors and showed high congruency to the respective array data (Table 3 ). The qRT-PCR data suggested considerable intergenic differences of absolute transcript abundances (Additional file 5).

\section{Comparison of transcriptomic responses to different types of stress}

We compared our microarray data to those of a study that was previously published for $F$. graminearum [10] to assess how many of the genes responding to tebuconazole would also respond to other types of stress. We applied the same statistical procedures described above to the published microarray data that examined two nutritional stress conditions, i.e. carbon and nitrogen starvation. Based on the $q$ value plots obtained by the EDGE software we used $q<0.01$ as the cut-off for statistical significance for these data sets. In addition, transcript levels in the published data needed to be changed at least twofold ( $\log _{2} \mathrm{FC} \geq 1$ and $\left.\leq-1\right)$ by the respective treatment to be considered significant. Resulting gene sets were used for subtractions (Figure 3). Transcript abundances from the majority, i.e. 457 (76.7\%) of the above described 596 genes were specifically increased after the fungicide treatment whereas 139 (23.3\%) genes responded also in at least one of the other two stress conditions (Figure $3 \mathrm{~A}$; Additional file 6). Some of those belonged to one of the three groups of proteins discussed above. FGSG_02346 (homolog of the S. cerevisiae gene Erg24), FGSG_09381 (homolog of S.c. Erg9) and FGSG_10424 (homolog of S.c. Erg19) belonged to the ergosterol biosynthesis pathway, whereas for ABC transporters and transcription factors we correspondingly identified three genes (FGSG_02786, FGSG_06771, FGSG_08312), and five genes (FGSG_01293, FGS G_01307, FGSG_01936, FGSG_06324, FGSG_10639), respectively. However, functional enrichment analysis with the remaining 457 genes whose transcripts were specifically increased by tebuconazole still indicated a statistically significant enrichment of genes involved in steroid metabolism (data not shown).

Comparison of the three stress conditions with respect to genes showing reduced transcript levels indicated considerably overlapping responses (Figure 3B). Of the 462 genes transcriptionally decreased after tebuconazole treatment $232(50.2 \%)$ were also decreased in at least one of the other two conditions. This included the genes FGSG_00069, FGSG_09177, FGSG_09333, FGSG_09349, FGSG_09852, FGSG_12970, FGSG_13172, all of which encode transcription factors. Importantly, none of the genes encoding enzymes of ergosterol biosynthesis or ABC transporters exhibited decreased transcript levels by the starvation stresses. Additional BlastP analyses focussing on those genes that were indicated to be specifically transcriptionally increased or decreased by the tebuconazole treatment revealed 42 , respectively 11 genes that appeared to be unique to $F$. graminearum 
A

$\begin{array}{cc}\begin{array}{c}\text { Carbon } \\ \text { starvation }\end{array} & \begin{array}{c}\text { Nitrogen } \\ \text { starvation }\end{array} \\ 1965 \text { genes } & 1431 \text { genes }\end{array}$

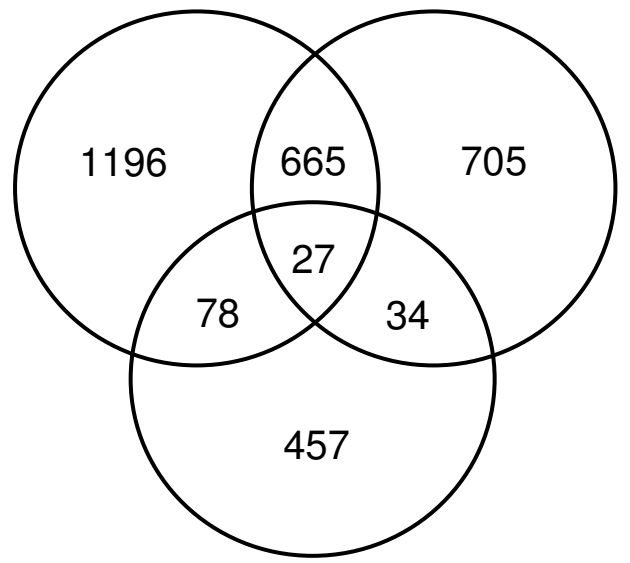

Tebuconazole

596 genes

B
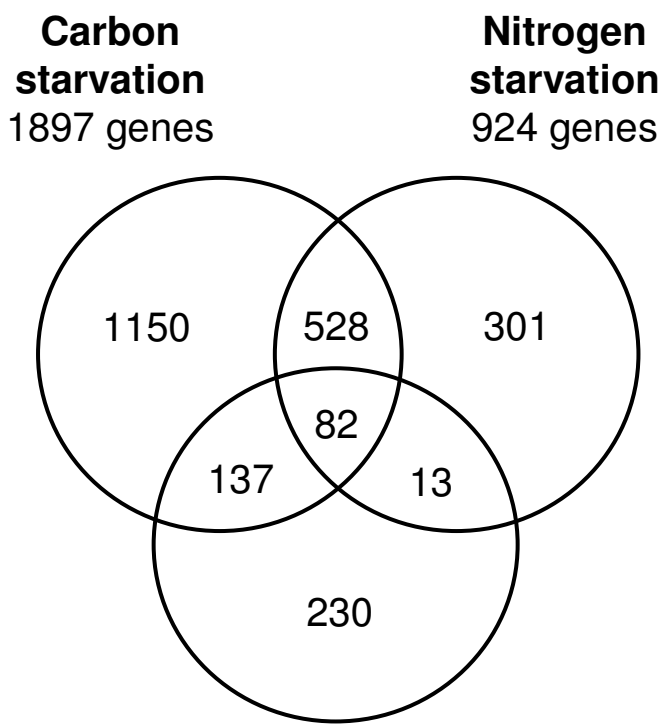

Tebuconazole 462 genes

Figure 3 Comparison of differentially expressed genes exposed to different stress conditions. Venn diagrams illustrate results from comparative analyses of gene sets found differentially expressed by azole treatment, carbon, or nitrogen starvation [10]. A. Comparisons of gene sets showing significantly increased transcript levels. Nine of 596 genes found in the current tebuconazole study did not match a probe set on the Affymetrix GeneChip. B. Comparisons of genes sets showing significantly decreased transcript levels. Three out of 462 genes did not match a probe set on the Affymetrix GeneChip. as they had no similarity at e $<10^{-10}$ to any other sequence in the current database (Additional file 6). Seventeen additional genes showing transcript levels specifically increased by tebuconazole found similar genes only in other Fusarium species (Additional file 6). The same applied to nine additional of the downregulated genes.

\section{Discussion}

In this study, we developed and validated a new $8 \times 15$ $\mathrm{k}$ Agilent microarray, and employed it to analyze gene expression in F. graminearum after treatment with tebuconazole. Our results demonstrate that this multiplex microarray is an effective and versatile tool to detect transcriptome responses at high sensitivity.

Due to its scientific and economic relevance, microarray technology has rapidly evolved into different platforms using short or long oligonucleotides provided by several commercial manufacturers, such as Affymetrix and Agilent Technologies. As studies using different microarray platforms indicated an overall good comparability [41], the choice of a platform is mainly governed by practical and cost considerations. Agilent's inkjet-like printing technology provides high flexibility for microarray design and allows convenient optimization in followup versions. In addition, multiplex formats were developed that carry several microarrays on a single slide, allowing for cost-effective transcription profiling. The chosen Agilent $8 \times 15 \mathrm{k}$ format is fully sufficient for covering the entire genome of F. graminearum, thus permitting to simultaneously perform eight independent expression profiling experiments. The employment of the one-color labeling technique recently introduced for the Agilent platform facilitates comparisons across microarrays and between groups of samples without compromising the quality of results [42].

To address the reliability of the results obtained by the new microarray, we selected 31 genes for determining their transcript levels by an independent experimental approach, i.e. qRT-PCR. For most of these genes, the $\log _{2} \mathrm{FC}$ values from the qRT-PCRs were very close to those of the microarray experiments. The comparison of the entire data sets reveals a highly positive correlation $(\mathrm{R}=0.95)$ which corroborates the results of a previous report that also examined these two experimental approaches [43].

The new $8 \times 15 \mathrm{k}$ Agilent microarray was used to analyze gene expression patterns in F. graminearum treated with tebuconazole. We assess the results in the context of microarray experiments that were performed earlier. On the one hand, results that are supported by those previously observed (e.g. in S. cerevisiae) highlight the capability of the new microarray to gain information relevant for better understanding the regulatory 
networks mediating azole responses. This will be especially helpful for studying novel fungicides whose modeof-action should not only be analyzed in a model organism like $S$. cerevisiae but ideally also in the targeted pathogens, e.g. F. graminearum. Consistent with studies in other fungi [44-47] we found that the transcript levels of most of the genes encoding proteins involved in ergosterol-biosynthesis were increased by azole treatment. Furthermore, our functional enrichment analyses using GeneOntology and FunCat demonstrated that the ergosterol pathway ranks at the top among all functional categories containing genes with significantly enhanced transcript abundances. Interestingly, we found that within this category FGSG_04092 (=Cyp51A), which is one of the three genes encoding CYP51 in F. graminearum, exhibited the most significantly increased transcript levels of the entire study. In S. cerevisiae binding of azoles to CYP51 leads to ergosterol depletion, accumulation of a toxic aberrant sterol and compromised membrane rigidity [48]. It is thus possible that a feed-back loop connecting transcriptional regulation of ergosterol biosynthesis and sterol levels exists in F. graminearum, as previously reported for $S$. cerevisiae [49]. In yeast this link is mediated by UPC2 and ECM11 which regulate transcription of sterol biosynthetic genes by binding to a sterol regulatory element (SRE) in their promotors [35].

The fact that our microarray study uncovered the transcript levels of genes in the ergosterol biosynthesis pathway as highly significantly increased by tebuconazole treatment demonstrates its utility for fungicide research. The data presented here clearly indicate that microarray analysis can contribute to identify unknown mode-of-actions, as has previously been shown for the fungicide ciclopirox olamine in Candida albicans [50]. Sixty percent of the up-regulated genes were found to encode proteins for iron uptake and metabolism suggesting that treated cells suffered from iron limitation. This was supported by physiological experiments showing that addition of $\mathrm{Fe}^{2+}$ or $\mathrm{Fe}^{3+}$ diminished the detrimental effect of ciclopirox olamine on germ tube formation.

Comparison of our results with published microarray data indicates that about one quarter of the genes upregulated by tebuconazole and almost half of the downregulated genes correspondingly responded when $F$. graminearum was exposed to two unrelated starvation stress conditions. This suggests that many of the genes exhibit a rather unspecific stress response, which may in part originate from defective nutrient supply resulting from the azole-mediated membrane perturbations. On the other hand, the majority of the genes with enhanced transcript abundances responded specifically to fungicide treatment, which is underlined by the fact that all
Cyp51 variants only responded in our azole treatment study. Thus, the novel microarray is an excellent tool supporting identification of target genes. Microarray data of course need to be confirmed in subsequent studies, e.g. by targeted gene deletion or RNAi experiments, to address the function of individual genes to attenuate fungicide impact and the mechanisms by which this may be achieved.

Currently it is uncertain whether the CYP51A, B, and $\mathrm{C}$ proteins have specific functions in $F$. graminearum. In Aspergillus fumigatus transformants carrying individual deletions of $C y p 51 A$ and $C y p 51 B$ remained viable whereas the gene family as a whole was essential [51]. However, in this human pathogen up to now only point mutations in Cyp51A but not in Cyp51B were discovered in clinical isolates and resistant strains generated in vitro [52-54]. This suggests that the functions of these proteins may not completely overlap.

Also ABC transporters were reported to be involved in azole stress responses and in the development of azole tolerance in several fungi as some of them mediate active efflux of fungicides and other xenobiotics [34]. The subfamily most closely associated with drug resistance in the Saccharomycotina is PDR which have up to 10 members in yeasts [55]. In contrast, the genome of F. graminearum harbors 19 PDR-type ABC transporters. An evolutionary expansion of the PDR subfamily was previously noticed for Pezizomycotina [56]. In addition, the numbers of genes encoding MRP and MDR transporters (16 each) are clearly increased in F. graminearum as compared to $S$. cerevisiae (4 and 7 , respectively).

Our microarray data provide valuable insight into the transcriptional responses of $\mathrm{ABC}$ transporter genes from F. graminearum, suggesting that several of them may attenuate the effects of tebuconazole action. Similarly, microarrays analyzing azole responses of C. albicans wild type strains showed that the transcript levels of the $\mathrm{ABC}$ transporter genes $C d r 1$ and $C d r 2$ were increased [46]. Both genes were also up-regulated in in vitro azole-adapted strains and in clinically resistant isolates $[57,58]$. Gene deletions in an azole-resistant isolate of C. albicans indicated that CDR1 is essential to mediate azole resistance whereas CDR2 seems to be less important [59]. However, heterologous overexpression of both $C d r 1$ or $C d r 2$ conferred increased azole tolerance to $S$. cerevisiae [60]. Another well-characterized ABC transporter is PDR5 of $S$. cerevisiae, which is exporting a wide range of xenobiotics, including azoles [60,61]. Additional reports underline the involvement of $\mathrm{ABC}$ transporters in contributing to fungicide resistance also in plant pathogenic Pezizomycotina $[62,63]$. Since most of this research has focused on PDR-type transporters, it will be interesting to analyze also the contribution of 
azole-responsive MDR- and MRP-type proteins in the future.

Transcription factors have been identified as additional elements in conferring fungicide resistance $[64,65]$. Since the corresponding transcriptional networks vary to some degree when comparing species in the Saccharomycotina $[64,66]$ it is important to extend such analyses to Pezizomycotina, including toxigenic plant pathogens like F. graminearum. Among the genes with significantly increased transcript levels, we detected several similarities with genes encoding the transcriptional regulators $U p c 2$ and Tac1 known to be involved in azole responses in S. cerevisiae and/or C. albicans. Also in C. albicans transcript levels of Upc2 and Tac1 increased in response to azole-stress [36,67]. In both yeasts, UPC2 homologs are activators of genes encoding proteins for ergosterol-biosynthesis and sterol uptake [35,67-69]. In C. albicans a disruption of Upc2 induced hypersusceptibility and its overexpression increased azole resistance [70]. A G648D exchange in UPC2 created an allele that constitutively up-regulated Erg11 expression and thereby improved azole resistance. Similarly, Tac1 in C. albicans and Pdr1 in S. cerevisiae regulate the PDR-type $\mathrm{ABC}$ transporter genes $C d r 1, C d r 2$, and $P d r 5$, respectively $[36,71]$. In these two yeasts transcription factors mediate the response to azoles by regulating the expression not only of genes for ergosterol biosynthesis but also of efflux transporters. Other putative regulators with significantly increased transcript levels were similar to CRZ1 from S. cerevisiae and C. albicans, a transcription factor that is involved in regulating cell wall integrity [37]. This may reflect a more general response of the fungus in surviving the fungicide treatment. Apart from putative transcription factors with similarity to proteins reported to coordinate azole stress responses in S. cerevisiae and C. albicans we found additional azole-responsive transcription factors in F. graminearum. The response of these factors may reflect differences in transcriptional regulation of azole response that are either specific for F. graminearum or higher taxonomic levels.

\section{Conclusions}

This study introduces a novel F. graminearum microarray that is flexible and cost effective due to its multiplex format. Gene expression analyses investigating the tebuconazole response indicated that this array when analyzed with proper bioinformatic tools will be very helpful in fungicide research, e.g. in elucidation of mechanisms of fungicide resistance or mode-of-action, and beyond. Detailed analyses of genes encoding ergosterol biosynthesis proteins, ABC transporters, and transcription factors established a base for functional studies of the F. graminearum regulatory network responding to azole exposure.

\section{Additional material}

\begin{abstract}
Additional file 1: Design of the $8 \times 15 \mathrm{k} F$. graminearum microarray This file includes information about gene-specific oligonucleotides and additional features assembled on the $15 \mathrm{k}$ microarray. In addition, the list of the corresponding transcript and gene IDs is provided for proper assignment of array oligonucleotides and data to a specific $F$. graminearum gene.

Additional file 2: Supplementary information about selected gene subsets. This file provides information and results about the analyzed genes encoding proteins for ergosterol biosynthesis, ABC transporters, and transcription factors. Beside statistical data of microarray experiments $\left(\log _{2}\right.$ FC, $q$ value, rank), information include BlastP results and gene descriptions for homologs in S. cerevisiae and C. albicans obtained from the databases SGD and CGD, and transcription factor classification data from FTFD.
\end{abstract}

Additional file 3: Statistical results of microarray hybridizations. This file includes information about statistical analyses with the software EDGE. Results shown include processed and normalized signal data used as input data and provides the original output data (Target ID, rank, a value, graphics). In addition, quantitative data of the fold-change (FC, $\log _{2}$ differences) are shown. Moreover, the file provides a complete list of genes with significantly increased resp. decreased transcript abundances.

Additional file 4: Functional enrichment analyses. This file includes results of analyses using the functional annotation databases GeneOntology and FunCat. Information is provided for protein functions determined as significantly enriched in sets of $F$. graminearum genes showing significantly altered transcript levels after tebuconazole treatment. Results are presented separately for the two databases as well as with respect to increased and decreased transcript levels.

Additional file 5: Comparison of transcript levels of selected genes as determined by qRT-PCR. The initial fluorescence $R_{0}$ is a relative measure for the abundance of transcripts. $R_{0}$ was calculated by application of sigmoidal curve-fitting.

Additional file 6: Supplementary data to Figure 3. This file provides detailed gene lists, the Venn diagrams in Figure 3 were based on.

\section{Acknowledgements}

This project was funded by the Deutsche Forschungsgemeinschaft and the Ministry of Education of the State of Saxony-Anhalt (PlantRessource II/TP3). BayerCropScience $\mathrm{GmbH}$ kindly provided the fungicide. The fungal strain was kindly provided by Gerhard Adam (BOKU, Vienna). We thank Martina Schad and Ralf Hennig (imaGenes, Berlin) for advice on statistics and many discussions. We thank Doris Jany (MLU Halle) for skilled technical assistance.

\section{Author details}

${ }^{1}$ Institut für Agrar- und Ernährungswissenschaften, Naturwissenschaftliche Fakultät III, Martin-Luther-Universität Halle-Wittenberg, Betty-Heimann-Str. 3, D-06120 Halle (Saale), Germany. ${ }^{2}$ Interdisziplinäres Zentrum für Nutzpflanzenforschung, Martin-Luther-Universität Halle-Wittenberg, BettyHeimann-Str. 3, D-06120 Halle (Saale), Germany.

\section{Authors' contributions}

RB contributed to conception of the study, collected and provided samples for microarray hybridizations, performed data analysis, initiated qRT-PCRs, and drafted the manuscript. FW conducted several qRT-PCR assays. HD was an advisor of the work and contributed to the manuscript. SW conceived and coordinated the project, and wrote the manuscript.

Received: 6 August 2010 Accepted: 21 January 2011

Published: 21 January 2011 


\section{References}

1. Osborne LE, Stein JM: Epidemiology of Fusarium head blight on smallgrain cereals. Int J Food Microbiol 2007, 119:103-108.

2. Cowger C, Patton-Ozkurt J, Brown-Guedira G, Perugini L: Post-anthesis moisture increased fusarium head blight and deoxynivalenol levels in North Carolina winter wheat. Phytopathology 2009, 99:320-327.

3. Rocha O, Ansari K, Doohan FM: Effects of trichothecene mycotoxins on eukaryotic cells: A review. Food Addit Contam 2005, 22:369-378.

4. Klix MB, Verreet JA, Beyer M: Comparison of the declining triazole sensitivity of Gibberella zeae and increased sensitivity achieved by advances in triazole fungicide development. Crop Prot 2007, 26:683-690

5. Yin Y, LiU X, Li B, Ma Z: Characterization of sterol demethylation inhibitorresistant isolates of Fusarium asiaticum and $F$. graminearum collected from wheat in China. Phytopathology 2009, 99:487-497.

6. Cools HJ, Fraaije BA: Are azole fungicides losing ground against Septoria wheat disease? Resistance mechanisms in Mycosphaerella graminicola. Pest Manag Sci 2008, 64:681-684.

7. Wyand RA, Brown JKM: Sequence variation in the CYP51 gene of Blumeria graminis associated with resistance to sterol demethylase inhibiting fungicides. Fungal Genet Biol 2005, 42:726-735.

8. Barker KS, Crisp S, Wiederhold N, Lewis RE, Bareither B, Eckstein J, Barbuch R, Bard M, Rogers PD: Genome-wide expression profiling reveals genes associated with amphotericin $B$ and fluconazole resistance in experimentally induced antifungal resistant isolates of Candida albicans. J Antimicrob Chemother 2004, 54:376-385.

9. Cuomo CA, Gueldener U, Xu JR, Trail F, Turgeon BG, Di Pietro A, Walton JD, Ma LJ, Baker SE, Rep M, et al: The Fusarium graminearum genome reveals a link between localized polymorphism and pathogen specialization. Science 2007, 317:1400-1402

10. Güldener U, Seong KY, Boddu J, Cho SH, Trail F, Xu JR, Adam G, Mewes HW, Muehlbauer GJ, Kistler HC: Development of a Fusarium graminearum Affymetrix GeneChip for profiling fungal gene expression in vitro and in planta. Fungal Genet Biol 2006, 43:316-325.

11. Relogio A, Schwager C, Richter A, Ansorge W, Valcarcel J: Optimization of oligonucleotide-based DNA microarrays. Nucleic Acids Res 2002, 30(11) e51.

12. Hughes TR, Mao M, Jones AR, Burchard J, Marton MJ, Shannon KW, Lefkowitz SM, Ziman M, Schelter JM, Meyer MR, et al: Expression profiling using microarrays fabricated by an ink-jet oligonucleotide synthesizer. Nat Biotechnol 2001, 19:342-347.

13. Becher R, Hettwer U, Karlovsky P, Deising HB, Wirsel SGR: Adaptation of Fusarium graminearum to tebuconazole yielded descendants diverging for levels of fitness, fungicide resistance, virulence, and mycotoxin production. Phytopathology 2010, 100:444-453

14. Correll JC, Klittich CJR, Leslie JF: Nitrate non-utilizing mutants of Fusarium oxysporum and their use in vegetative compatibility tests. Phytopathology 1987, 77:1640-1646.

15. Krijger JJ, Horbach R, Behr M, Schweizer P, Deising HB, Wirsel SGR: The yeast signal sequence trap identifies secreted proteins of the hemibiotrophic corn pathogen Colletotrichum graminicola. Mol Plant Microbe Interact 2008, 21:1325-1336.

16. Irizarry RA, Hobbs B, Collin F, Beazer-Barclay YD, Antonellis KJ, Scherf U, Speed TP: Exploration, normalization, and summaries of high density oligonucleotide array probe level data. Biostatistics 2003, 4:249-264.

17. Bolstad BM, Irizarry RA, Astrand M, Speed TP: A comparison of normalization methods for high density oligonucleotide array data based on variance and bias. Bioinformatics 2003, 19:185-193.

18. Storey JD, Dai JY, Leek JT: The optimal discovery procedure for largescale significance testing, with applications to comparative microarray experiments. Biostatistics 2007, 8:414-432.

19. Leek JT, Monsen E, Dabney AR, Storey JD: EDGE: extraction and analysis of differential gene expression. Bioinformatics 2006, 22:507-508.

20. Storey JD, Tibshirani R: Statistical significance for genomewide studies. Proc Natl Acad Sci USA 2003, 100:9440-9445

21. Rüpp A, Zollner A, Maier D, Albermann K, Hani J, Mokrejs M, Tetko I, Guldener U, Mannhaupt G, Munsterkotter M, et al: The FunCat, a functional annotation scheme for systematic classification of proteins from whole genomes. Nucleic Acids Res 2004, 32:5539-5545.

22. Ashburner M, Ball CA, Blake JA, Botstein D, Butler H, Cherry JM, Davis AP, Dolinski K, Dwight SS, Eppig JT, et al: Gene Ontology: tool for the unification of biology. Nat Genet 2000, 25:25-29.
23. Conesa A, Gotz S, Garcia-Gomez JM, Terol J, Talon M, Robles M: Blast2GO: a universal tool for annotation, visualization and analysis in functional genomics research. Bioinformatics 2005, 21:3674-3676.

24. Benjamini $Y$, Hochberg $Y$ : Controlling the false discovery rate - a practical and powerful approach to multiple testing. J R Stat Soc Ser B-Methodol 1995, 57:289-300

25. Daum G, Lees ND, Bard M, Dickson R: Biochemistry, cell biology and molecular biology of lipids of Saccharomyces cerevisiae. Yeast 1998, 14:1471-1510.

26. Gaur M, Choudhury D, Prasad R: Complete inventory of $A B C$ proteins in human pathogenic yeast, Candida albicans. J Mol Microbiol Biotechnol 2005, 9:3-15

27. Bauer $B E$, Wolfger $H$, Kuchler $K$ : Inventory and function of yeast $A B C$ proteins: about sex, stress, pleiotropic drug and heavy metal resistance. Biochim Biophys Acta 1999, 1461:217-236.

28. Tamura K, Dudley J, Nei M, Kumar S: MEGA4: Molecular evolutionary genetics analysis (MEGA) software version 4.0. Mol Biol Evol 2007, 24:1596-1599.

29. Liu WH, Saint DA: A new quantitative method of real time reverse transcription polymerase chain reaction assay based on simulation of polymerase chain reaction kinetics. Anal Biochem 2002, 302:52-59.

30. Vandesompele J, De Preter K, Pattyn F, Poppe B, Van Roy N, De Paepe A, Speleman F: Accurate normalization of real-time quantitative RT-PCR data by geometric averaging of multiple internal control genes. Genome Biol 2002, 3:0034.0031-0034.0011.

31. Ferreira ME, Colombo AL, Paulsen I, Ren Q, Wortman J, Huang J, Goldman MH, Goldman GH: The ergosterol biosynthesis pathway, transporter genes, and azole resistance in Aspergillus fumigatus. Med Mycol 2005, 43:S313-319.

32. Mellado E, Diaz-Guerra TM, Cuenca-Estrella M, Rodriguez-Tudela JL: Identification of two different 14-alpha sterol demethylase-related genes (cyp51A and cyp51B) in Aspergillus fumigatus and other Aspergillus species. J Clin Microbiol 2001, 39:2431-2438.

33. Liu WH, Saint DA: Validation of a quantitative method for real time PCR kinetics. Biochem Biophys Res Commun 2002, 294:347-353.

34. De Waard MA, Andrade AC, Hayashi K, Schoonbeek HJ, Stergiopoulos I, Zwiers LH: Impact of fungal drug transporters on fungicide sensitivity, multidrug resistance and virulence. Pest Manag Sci 2006, 62:195-207.

35. Vik A, Rine J: Upc2p and Ecm22p, dual regulators of sterol biosynthesis in Saccharomyces cerevisiae. Mol Cell Biol 2001, 21:6395-6405.

36. Coste AT, Karababa M, Ischer F, Bille J, Sanglard D: TAC1, transcriptional activator of $C D R$ genes, is a new transcription factor involved in the regulation of Candida albicans $A B C$ transporters CDR1 and CDR2. Eukaryot Cell 2004, 3:1639-1652

37. Matheos DP, Kingsbury TJ, Ahsan US, Cunningham KW: Tcn1p/Crz1p, a calcineurin-dependent transcription factor that differentially regulates gene expression in Saccharomyces cerevisiae. Genes Dev 1997 11:3445-3458

38. Hellauer K, Akache B, MacPherson S, Sirard E, Turcotte B: Zinc cluster protein Rdr1p Is a transcriptional repressor of the PDR5 gene encoding a multidrug transporter. J Biol Chem 2002, 277:17671-17676.

39. Castrejon F, Gomez A, Sanz M, Duran A, Roncero C: The RIM101 pathway contributes to yeast cell wall assembly and its function becomes essential in the absence of mitogen-activated protein kinase Slt2p. Eukaryot Cell 2006, 5:507-517

40. Ikeda M, Kihara A, Denpoh A, Igarashi Y: The Rim101 pathway is involved in Rsb1 expression induced by altered lipid asymmetry. Mol Biol Cell 2008, 19:1922-1931.

41. Li ZG, Su ZQ, Wen ZN, Shi LM, Chen T: Microarray platform consistency is revealed by biologically functional analysis of gene expression profiles. BMC Bioinformatics 2009, 10 Suppl 11:S12

42. Patterson TA, Lobenhofer EK, Fulmer-Smentek SB, Collins PJ, Chu TM, Bao WJ, Fang $H$, Kawasaki ES, Hager J, Tikhonova IR, et al: Performance comparison of one-color and two-color platforms within the MicroArray Quality Control (MAQC) project. Nat Biotechnol 2006, 24:1140-1150.

43. Dallas PB, Gottardo NG, Firth MJ, Beesley AH, Hoffmann K, Terry PA, Freitas JR, Boag JM, Cummings AJ, Kees UR: Gene expression levels assessed by oligonucleotide microarray analysis and quantitative realtime RT-PCR - how well do they correlate? BMC Genomics 2005, 6(1):59.

44. Bammert GF, Fostel JM: Genome-wide expression patterns in Saccharomyces cerevisiae: Comparison of drug treatments and genetic 
alterations affecting biosynthesis of ergosterol. Antimicrob Agents Chemother 2000, 44:1255-1265.

45. Agarwal AK, Rogers PD, Baerson SR, Jacob MR, Barker KS, Cleary JD, Walker LA, Nagle DG, Clark AM: Genome-wide expression profiling of the response to polyene, pyrimidine, azole, and echinocandin antifungal agents in Saccharomyces cerevisiae. J Biol Chem 2003, 278:34998-35015.

46. Liu TT, Lee REB, Barker KS, Lee RE, Wei L, Homayouni R, Rogers PD: Genome-wide expression profiling of the response to azole, polyene, echinocandin, and pyrimidine antifungal agents in Candida albicans. Antimicrob Agents Chemother 2005, 49:2226-2236.

47. Diao YJ, Zhao R, Deng XM, Leng WC, Peng JP, Jin Q: Transcriptional profiles of Trichophyton rubrum in response to itraconazole. Med Mycol 2009, 47:237-247.

48. Abe F, Usui K, Hiraki T: Fluconazole modulates membrane rigidity, heterogeneity, and water penetration into the plasma membrane in Saccharomyces cerevisiae. Biochemistry 2009, 48:8494-8504.

49. Smith SJ, Crowley JH, Parks LW: Transcriptional regulation by ergosterol in the yeast Saccharomyces cerevisiae. Mol Cell Biol 1996, 16:5427-5432.

50. Sigle HC, Thewes S, Niewerth M, Korting HC, Schäfer-Korting M, Hube B: Oxygen accessibility and iron levels are critical factors for the antifungal action of ciclopirox against Candida albicans. J Antimicrob Chemother 2005, 55:663-673.

51. Hu WQ, Sillaots S, Lemieux S, Davison J, Kauffman S, Breton A, Linteau A, Xin $C L$, Bowman J, Becker J, et al: Essential gene identification and drug target prioritization in Aspergillus fumigatus. PLoS Pathog 2007, 3(3):e24.

52. Mann PA, Parmegiani RM, Wei SQ, Mendrick CA, Li X, Loebenberg D, DiDomenico B, Hare RS, Walker SS, McNicholas PA: Mutations in Aspergillus fumigatus resulting in reduced susceptibility to posaconazole appear to be restricted to a single amino acid in the cytochrome p450 14 alphademethylase. Antimicrob Agents Chemother 2003, 47:577-581.

53. Mellado E, Garcia-Effron G, Alcazar-Fuoli L, Melchers WJG, Verweij PE, Cuenca-Estrella A, Rodriguez-Tudela JL: A new Aspergillus fumigatus resistance mechanism conferring in vitro cross-resistance to azole antifungals involves a combination of cyp51A alterations. Antimicrob Agents Chemother 2007, 51:1897-1904

54. Snelders E, van der Lee HA, Kuijpers J, Rijs AJ, Varga J, Samson RA, Mellado E, Donders AR, Melchers WJ, Verweij PE: Emergence of azole resistance in Aspergillus fumigatus and spread of a single resistance mechanism. PloS Med 2008, 5:e219.

55. Cannon RD, Lamping E, Holmes AR, Niimi K, Baret PV, Keniya MV, Tanabe K, Niimi M, Goffeau A, Monk BC: Efflux-mediated antifungal drug resistance. Clin Microbiol Rev 2009, 22:291-321.

56. Kovalchuk A, Driessen AJ: Phylogenetic analysis of fungal $A B C$ transporters. BMC Genomics 2010, 11:177.

57. Cowen LE, Sanglard D, Calabrese D, Sirjusingh C, Anderson JB, Kohn LM: Evolution of drug resistance in experimental populations of Candida albicans. J Bacteriol 2000, 182:1515-1522.

58. Sanglard D, Kuchler K, Ischer F, Pagani JL, Monod M, Bille J: Mechanisms of resistance to azole antifungal agents in Candida albicans isolates from AIDS patients involve specific multidrug transporters. Antimicrob Agents Chemother 1995, 39:2378-2386.

59. Tsao S, Rahkhoodaee F, Raymond M: Relative contributions of the Candida albicans ABC transporters Cdr1p and Cdr2p to clinical azole resistance. Antimicrob Agents Chemother 2009, 53:1344-1352.

60. Lamping E, Monk BC, Niimi K, Holmes AR, Tsao S, Tanabe K, Niimi M, Uehara $Y$, Cannon RD: Characterization of three classes of membrane proteins involved in fungal azole resistance by functional hyperexpression in Saccharomyces cerevisiaev. Eukaryot Cell 2007, 6:1150-1165.

61. Kolaczkowski M, vanderRest M, CybularzKolaczkowska A, Soumillion JP, Konings WN, Goffeau A: Drugs, ionophoric peptides, and steroids as substrates of the yeast multidrug transporter Pdr5p. J Biol Chem 1996, 271:31543-31548.

62. Zwiers LH, Stergiopoulos L, Van Nistelrooy JGM, De Waard MA: ABC transporters and azole susceptibility in laboratory strains of the wheat pathogen Mycosphaerella graminicola. Antimicrob Agents Chemother 2002, 46:3900-3906.

63. Hayashi K, Schoonbeek HJ, Sugiura H, De Waard MA: Multidrug resistance in Botrytis cinerea associated with decreased accumulation of the azole fungicide oxpoconazole and increased transcription of the $A B C$ transporter gene BcatrD. Pestic Biochem Physiol 2001, 70:168-179.
64. Sanglard D, Coste A, Ferrari S: Antifungal drug resistance mechanisms in fungal pathogens from the perspective of transcriptional gene regulation. FEMS Yeast Res 2009, 9:1029-1050.

65. Monk BC, Goffeau A: Outwitting multidrug resistance to antifungals. Science 2008, 321:367-369.

66. Lelandais G, Tanty V, Geneix C, Etchebest C, Jacq C, Devaux F: Genome adaptation to chemical stress: clues from comparative transcriptomics in Saccharomyces cerevisiae and Candida glabrata. Genome Biol 2008, 9(11): R164.

67. MacPherson S, Akache B, Weber S, De Deken X, Raymond M, Turcotte B: Candida albicans zinc cluster protein Upc $2 p$ confers resistance to antifungal drugs and is an activator of ergosterol biosynthetic genes. Antimicrob Agents Chemother 2005, 49:1745-1752.

68. Wilcox LJ, Balderes DA, Wharton B, Tinkelenberg AH, Rao G, Sturley SL: Transcriptional profiling identifies two members of the ATP-binding cassette transporter superfamily required for sterol uptake in yeast. J Biol Chem 2002, 277:32466-32472.

69. Silver PM, Oliver BG, White TC: Role of Candida albicans transcription factor Upc2p in drug resistance and sterol metabolism. Eukaryot Cell 2004, 3:1391-1397.

70. Dunkel N, Liu TT, Barker KS, Homayouni R, Morschhauser J, Rogers PD: A gain-of-function mutation in the transcription factor Upc2p causes upregulation of ergosterol biosynthesis genes and increased fluconazole resistance in a clinical Candida albicans isolate. Eukaryot Cell 2008, 7:1180-1190

71. Gao C, Wang LM, Milgrom E, Shen WCW: On the mechanism of constitutive Pdr1 activator-mediated PDR5 transcription in Saccharomyces cerevisiae - Evidence for enhanced recruitment of coactivators and altered nucleosome structures. J Biol Chem 2004 279:42677-42686.

doi:10.1186/1471-2164-12-52

Cite this article as: Becher et al: Development of a novel multiplex DNA microarray for Fusarium graminearum and analysis of azole fungicide responses. BMC Genomics 2011 12:52.

\section{Submit your next manuscript to BioMed Central and take full advantage of:}

- Convenient online submission

- Thorough peer review

- No space constraints or color figure charges

- Immediate publication on acceptance

- Inclusion in PubMed, CAS, Scopus and Google Scholar

- Research which is freely available for redistribution 